OPEN ACCESS

Edited by:

Yanhui Liao,

Sir Run Run Shaw Hospital, China

Reviewed by:

Yawen Sun

Shanghai JiaoTong University, China

Feng Liu,

Tianjin Medical University General

Hospital, China

*Correspondence:

Dahua Yu

fmydh@imust.edu.cn

Haitao Ju

835798312@qq.com

Kai Yuan

kyuan@xidian.edu.cn

Specialty section: This article was submitted to

Addictive Disorders,

a section of the journal

Frontiers in Psychiatry

Received: 08 May 2020

Accepted: 26 June 2020

Published: 10 July 2020

Citation:

Xue T, Dong F, Huang R, Tao $Z$, Tang J, Cheng Y, Zhou M, Hu Y, Li X, Yu D, Ju H and Yuan K (2020) Dynamic Neuroimaging Biomarkers of Smoking in Young Smokers.

Front. Psychiatry 11:663. doi: 10.3389/fpsyt.2020.00663

\section{Dynamic Neuroimaging Biomarkers of Smoking in Young Smokers}

\author{
Ting Xue ${ }^{1,2}$, Fang Dong ${ }^{2}$, Ruoyan Huang ${ }^{2}$, Zhanlong Tao $^{1}$, Jun Tang ${ }^{1}$, Yongxin Cheng ${ }^{2}$, \\ Mi Zhou ${ }^{2}$, Yiting $\mathrm{Hu}^{2}$, Xiaojian $\mathrm{Li}^{2}$, Dahua $\mathrm{Yu}^{2 *}$, Haitao $\mathrm{Ju}^{3 *}$ and Kai Yuan ${ }^{2,4 *}$ \\ 1 School of Science, Inner Mongolia University of Science and Technology, Baotou, China, 2 Inner Mongolia Key Laboratory \\ of Pattern Recognition and Intelligent Image Processing, School of Information Engineering, Inner Mongolia University of \\ Science and Technology, Baotou, China, ${ }^{3}$ Department of Neurosurgery, Affiliated Hospital of Inner Mongolia Medical \\ University, Hohhot, China, ${ }^{4}$ Life Sciences Research Center, School of Life Science and Technology, Xidian University, \\ Xi'an, China
}

Objective: To examine potential changes in the dynamic characteristics of regional neural activity in young smokers and to detect whether the changes were associated with smoking behavior.

Methods: The dynamic regional homogeneity (dReHo) and dynamic amplitude of lowfrequency fluctuations (dALFF) in 40 young smokers and 42 nonsmokers were compared. Correlation analyses were also performed between dReHo and dALFF in areas showing group differences and smoking behavior [e.g., the Fagerström Test for Nicotine dependence (FTND) scores and pack-years].

Results: Significantly differences in dReHo variability were observed in the inferior frontal gyrus (IFG), superior frontal gyrus (SFG), medial frontal gyrus (MFG), insula, cuneus, postcentral gyrus, inferior semi-lunar lobule, orbitofrontal gyrus, and inferior temporal gyrus (ITG). Young smokers also showed significantly increased dALFF variability in the anterior cingulate cortex (ACC) and ITG. Furthermore, a significant positive correlation was found between dALFF variability in the ACC and the pack-years; whereas a significant negative correlation between dReHo variability in the IFG and the FTND scores was found in young smokers.

Conclusion: The pattern of resting state regional neural activity variability was different between young smokers and nonsmokers. Dynamic regional indexes might be a novel neuroimaging biomarker of smoking behavior in young smokers.

Keywords: dynamic, amplitude of low-frequency fluctuation (ALFF), young smokers, resting-state functional magnetic resonance imaging (rs-fMRI), regional homogeneity (ReHo)

\section{INTRODUCTION}

There are more than one billion smokers globally according to the report in 2017 published by the Word Health Organization (https://www.who.int/). It has been demonstrated that the age period from late adolescence to adulthood is a crucial period of time of continued brain development (1). Numerous findings showed that people at this age have high prevalence of smoking cigarettes, and people who start smoking cigarettes at this age is more susceptible to nicotine addiction $(2,3)$. 
Therefore, the elucidation of neural mechanisms underlying smoking cigarettes in young smokers might be helpful for preventing nicotine addiction.

Although the exact mechanism of nicotine addiction is still unknown, recent neuroimaging findings have advanced our understanding (4-8). Resting-state functional magnetic resonance imaging ( $r s-f M R I)$, measuring task-independent spontaneous brain activity in low-frequency blood oxygenation level dependent (BOLD) fluctuations (9), has been widely applied for delineating functional neural abnormalities in smokers (10-17). Previous studies have demonstrated that regional spontaneous neural characteristics are very important for understanding neuropathological and neurophysiological conditions (18). For example, abnormal regional energy consumption indicates decreased or excessive resting metabolic rates (19). Currently, the widely used approaches for characterizing spontaneous regional neural properties are the regional homogeneity ( $\mathrm{ReHo}$ ) and amplitude of low-frequency fluctuation (ALFF). Specifically, ReHo measures the temporal synchronization of regional neural activity among spatially adjacent regions (20); whereas ALFF reflects the intensity of regional neural activity (21). These two approaches have been widely applied to evaluate regional neural function in neuropsychiatric diseases and neurologic disorders (21-24).

In the context of nicotine addiction, numerous rs-fMRI studies have focused on the properties of regional neural activity (12, 25-28). For example, compared with nonsmokers, $\mathrm{Yu}$ and his colleague discovered that heavy smokers exhibited decreased ReHo in the prefrontal regions and increased ReHo in the insula and posterior cingulate cortex (PCC) (12). Another study found decreased ReHo in the inferior frontal cortex and increased ReHo in the superior parietal lobe (25). Chen et al. further found decreased ReHo in the superior frontal gyrus (SFG) and precuneus, and the decreased ReHo value in the precuneus was positively correlated with the Fagerström Test for Nicotine dependence (FTND) scores, an important index for clinical evaluation of smoker's dependence on nicotine addiction (26). Using fractional ALFF technology, enhanced neural spontaneous activity was found in the caudate of young smokers (28). Another study focused on regional spontaneous neural activity intensity in heavy smoker and discovered decreased spontaneous activity in the precuneus and inferior temporal gyrus (ITG) and increased spontaneous activity in the caudate (27). These findings indicated that static ReHo and ALFF were different at rest between smokers and nonsmokers.

However, the above-mentioned investigations ignored the dynamic characteristics of spontaneous neural activity, and they usually assumed the BOLD signal during scanning was stationary. In fact, it has been suggested that the brain dynamically responds and adjusts to external or internal stimuli over multiple timescales $(29,30)$. By characterizing temporal changes in the brain's resting state functional connectivity, the intrinsic neural activity was also found to be time-varying in nature (31-34). Moreover, high-resolution spatiotemporal functional neuroimaging studies have confirmed substantial temporal variations in regional neural activity $(35,36)$. Consequently, elucidation of the temporal variability in oscillation amplitudes and synchronization of regional neural activity may help us better understand the effects of smoking cigarettes on brain function. Fortunately, the time-varying characteristics of resting state regional neural activity may be examined by the sliding window approach. This approach has been shown to work well for evaluating dynamic properties of regional neural activity (37), and it has been widely applied due to its simplicity and ease of implementation $(30,38)$.

Taken together, considering there are substantial temporal fluctuations in regional neural activity (35), and until now, there have been very few studies concerned with the dynamic characteristics of regional neural activity in young smokers. Currently, a sliding window technology combined with the dynamic ALFF (dALFF) and dynamic ReHo (dReHo) approaches were applied to investigate whether young smokers would present with abnormal dynamic characteristics of regional neural activity. In addition, correlation analyses were also performed to ascertain the relationships between the altered regional indexes (the dALFF and $\mathrm{dReHo}$ ) and smoking behavior. We hypothesized that the temporal characteristics of regional neural activity may be altered in young smokers, and dynamic indexes may be associated with smoking behavior. It is hoped that the present study advances our understanding of nicotine addiction.

\section{MATERIALS AND METHODS}

\section{Ethics Statement}

The present study was approved by the Ethical Committee of Medical Research in First Affiliated Hospital of Baotou Medical College, Inner Mongolia University of Science and Technology. All study procedures were conducted in accordance with the Declaration of Helsinki. After explaining the aims and steps in the procedure of the current study, all the participants and their guardians signed written informed consents.

\section{Subjects}

The potential participants were male undergraduates from local universities recruited through advertisements. To screen for potential participants, a semistructured interview and questionnaires were used to assess psychiatric conditions, medical conditions, medication use, history of claustrophobia, history of substance use, and the presence of metal implants in their body. The inclusion criteria for smokers were 1) meeting DSM-V criteria for nicotine dependence, 2) reporting smoking $\geq 10$ cigarettes per day in the last 6 months, 3 ) carbon monoxide $(\mathrm{CO})>6$ parts per million (p.p.m.) in expired air (Smokelyzer, Bedfont Scientific, Kent, UK), and 4) having no period of smoking abstinence longer than 6 months in the past few years. We used the FTND and pack-years to assess nicotine dependence (39). Specifically, the FTND measured the severity of nicotine dependence (40), and a pack-years was defined as twenty cigarettes smoked per day for one year. The nonsmokers were included as those who smoked less than five cigarettes in their lifetime. Additional requirements for nonsmokers were $\mathrm{CO}$ concentrations below 3 p.p.m. in expired air. Exclusion criteria for 
both groups were 1) any physical illness such as epilepsy, brain tumor, obstructive lung disease, or hepatitis, 2) urine test revealing any other substance or drug abuse (except nicotine), 3) existence of a neurological disease, or 4) claustrophobia. A total of 520 potential subjects completed the semi-structured interview and questionnaires, and among them, 46 participants were recruited in the young smoker group. The incidence was $8.8 \%$. Forty-eight nonsmokers were recruited in healthy controls group. All participants were righthanded and 17-24 years of age. This study only focused on differences in regional neural activity between nondeprived young smokers and nonsmokers. Therefore, young smokers were asked to refrain from smoking in the $30 \mathrm{~min}$ immediately preceding the scan to exclude withdrawal symptoms (41).

\section{Data Acquisition}

The study was performed on a $3 \mathrm{~T}$ magnetic resonance imaging (MRI) scanner (Achieva, Philips Healthcare, Best, the Netherlands) equipped with an 8-channel SENSE coil. The scanning parameters were repetition time $(\mathrm{TR})=2,000 \mathrm{~ms}$, echo time $(\mathrm{TE})=30 \mathrm{~ms}$, slice thickness $=5 \mathrm{~mm}$, number of slices $=30$, flip angle $=90^{\circ}$, field of view $($ FOV $)=220 \mathrm{~mm} \times$ $220 \mathrm{~mm}$, voxel size $=3 \mathrm{~mm} \times 3 \mathrm{~mm} \times 3 \mathrm{~mm}$, matrix $=64 \times 64$, and 180 volumes. During the entire scanning procedure, a standard head coil and foam pads were used to minimize head movement. All subjects were instructed to close their eyes and stay still during scanning.

\section{Data Analysis}

The rs-fMRI data were preprocessed using the Data Processing Assistant for Resting-State fMRI Analysis Toolkit (DPARSF). The details included 1) deleting the first 10 images, 2) correcting slice-time delay, 3) realigning to correct for head motion, 4) normalizing to the standard Montreal Neurological Institute (MNI) template provided by SPM using linear and nonlinear transformations, 5) resampling ( $3 \mathrm{~mm} \times 3 \mathrm{~mm} \times 3 \mathrm{~mm})$, and 6) regressing out nuisance covariates ( 24 head motion parameters, white matter signal, cerebrospinal fluid signal and linear trend). The head motion of all participants was examined. If the head motion displacement exceeded $2.0 \mathrm{~mm}$ or rotation exceed $2.0^{\circ}$, the corresponding data will be excluded from the current study. Six nonsmokers and six young smokers were excluded because of excessive head motion. Eventually, 40 young smokers and 42 nonsmokers were included in further analysis. Group differences in the mean framewise displacement (FD) were also compared. The results showed that there were no significant group differences ( $p=0.781)$ between the two group groups. Additionally, each participant's mean FD was included as a covariate in the follow-up, group-level analysis to reduce the effect of head movement. For ReHo calculations, the data were further filtered using a bandpass temporal filter of $0.01-0.08 \mathrm{~Hz}$ to reduce noise and low frequency drift.

The dALFF and dReHo analyses were performed using Temporal Dynamic Analysis (TDA) toolkits based on DPABI (42). Specifically, a temporal rectangular window was first chosen. Then, the ALFF and ReHo values in each window were calculated. Previous window-based analyses usually adopted a window length as small as $10 \mathrm{~s}$ (43) and as long as
$180 \mathrm{~s}(44,45)$. Ideally, the window size should be small enough to detect potentially transient signals, and yet large enough to analyze the lowest frequencies of interest in signals $(45,46)$. To avoid the introduction of spurious fluctuations, the minimum window length should be larger than $1 / f_{\min }$, where $f_{\min }$ is the minimum frequency of time series (47). Since there was currently no formal consensus regarding the window length, here a window length of 32 TR was selected according to previous studies (45), which was considered as the optimal parameter to maintain the balance between capturing a rapidly shifting dynamic temporal characteristics and obtaining reliable estimates of the regional neural activity (48). The preprocessed data of each individual were segmented into 139 windows. The ALFF and ReHo indexes were calculated in each window. In detail, the ReHo index was calculated using the Kendall's coefficient of concordance (KCC) to characterize the similarity of the time series of a given voxel and its 27 neighboring voxels (20). The ALFF index was calculated as the amplitude interval over a frequency range of $0.01-0.08 \mathrm{~Hz}$, reflecting the intensity of spontaneous neural activity (21). To obtain the dALFF and dReHo maps, the standard deviation (SD) of ReHo and ALFF values at each voxel across all windows were calculated. Then, the obtained dReHo and dALFF maps were further normalized by dividing dReHo and dALFF maps by the mean dReHo and dALFF values. Finally, an isotropic Gaussian kernel of $4 \mathrm{~mm}$ full-width-athalf-maximum (FWHM) was used to smooth the maps.

\section{Statistical Analysis}

Group comparisons of demographic data were performed using two-sample $t$ tests $(P<0.05)$. Group analyses of dALFF and $\mathrm{dReHo}$ maps were performed using the general linear model (GLM) in SPM12 with mean FD as a covariate. The results were thresholded at $P<0.001$ (uncorrected) and a cluster size of 50 voxels, corresponding to a familywise error (FWE) correction for multiple comparisons of $P_{\mathrm{FWE}}<0.05$. Correlation analyses were conducted in young smokers between smoking behavior (FTND and pack-years) and mean $\mathrm{dReHo/dALFF}$ values in regions showing group differences with mean FD as a covariate. In the current study, the potential relationship between dynamic regional indexes and smoking behavior were surveyed in an exploratory investigation. Therefore, the threshold for statistical significance was set at $P<0.05$ (uncorrected).

\section{RESULTS}

\section{Smoking Behavior}

Forty young male smokers and 42 male nonsmokers were included in the current study. The detailed demographic information was presented in Table 1.

\section{Group Differences in dReHo and dALFF Maps}

Compared with nonsmokers, young smokers demonstrated significantly decreased dReHo variability in the inferior frontal gyrus (IFG), medial frontal gyrus (MFG), SFG, inferior semilunar lobule, postcentral gyrus, cuneus, and insula, whereas significantly increased dReHo variability was observed in the 
TABLE 1 | Demographic and smoking behavior.

\begin{tabular}{lcc}
\hline & Young smokers $(\boldsymbol{n}=\mathbf{4 0})$ & Nonsmokers $(\boldsymbol{n}=\mathbf{4 2})$ \\
\hline Age, years & $19.70 \pm 2.02$ & $19.05 \pm 1.72$ \\
sex(M/F) & $40 / 0$ & $42 / 0$ \\
FTND & $6.73 \pm 1.48$ & - \\
pack-years & $2.44 \pm 0.93$ & - \\
\hline
\end{tabular}

Data represent mean \pm standard deviation; $M$, male; $F$, female; FTND, Fagerström Test for Nicotine Dependence; Pack-years, Years of smoking $\times$ Cigarettes smoked per day/20.

orbitofrontal gyrus and ITG (Figure 1 and Table 2). Relative to the nonsmoker group, young smokers showed significantly increased dALFF variability in the ITG and anterior cingulate cortex (ACC) (Figure 1 and Table 3).

\section{Correlation Analysis}

The results showed that the temporal variability in ALFF in the ACC was positively correlated with pack-years $(r=0.3268, p=0.0396$, uncorrected; Figure 2A), and the temporal variability in dReHo in the IFG was negatively correlated with FTND $(r=-0.3147, p=0.0479$, uncorrected; Figure 2B).

\section{DISCUSSION}

Previous studies have probed static regional neural activity in smokers. However, to the best of our knowledge, very few studies have tried to assess nicotine-related dynamic regional neural activity changes in young smokers. In the current study, dynamic regional neural activity between young smokers and nonsmokers was examined using sliding window analysis. Differences in dReHo variability were observed in the IFG, SFG, MFG, insula, cuneus, postcentral gyrus, inferior semi-lunar lobule, orbitofrontal gyrus, and ITG, whereas differences in dALFF variability in the ACC and ITG distinguished young smokers from nonsmokers. Further correlation analyses showed that the pack-years/FTND scores were related to abnormal dALFF variability in ACC/dReHo variability in IFG in young smokers. The current findings about the dynamic neural characteristics in young smokers may expand our understanding of nicotinerelated neural mechanisms.

The aberrant reward function is believed to play a key role in the progress of addiction. To date, the findings in this field showed that subcortical regions are crucial to facilitating such progress $(49,50)$. However, recent studies indicated that the frontal-cingulate regions are also heavily involved in addiction progress (51). For example, the ACC was found to be hyperactivated to drug-related reward and hypoactivated to natural rewards $(52,53)$. Recent studies further confirmed the function of ACC linking reward to goal-directed behaviors (54). One study focused on substance dependence found that compared with drug-related stimuli, substance-dependent individual exhibit decreased reward to stimuli related to

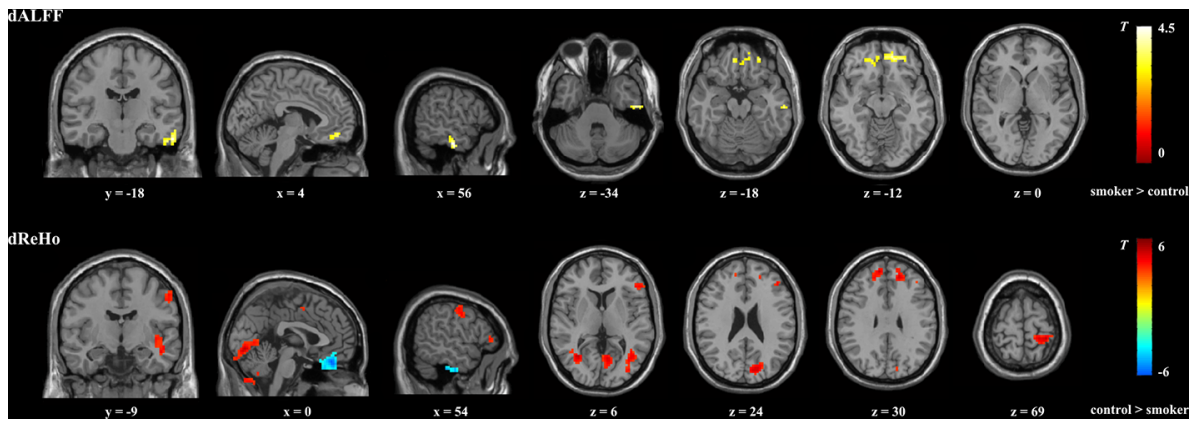

FIGURE 1 | Brain regions showing significant group differences in dALFF and dReHo between young smokers and nonsmokers using familywise error-corrected analysis ( $P_{F W E}<0.05$ with a minimum cluster size of $k=50$ voxels and a cluster-defining threshold $\left.P<0.001\right)$. dALFF, dynamic amplitude of low-frequencey fluctuation; dReHo, dynamic regional homogeneity.

TABLE 2 | Brain regions showing significant differences in dynamic ReHo between groups.

\begin{tabular}{|c|c|c|c|c|c|c|c|}
\hline & \multirow[b]{2}{*}{ Brain region } & \multirow[b]{2}{*}{ BA } & \multicolumn{3}{|c|}{ MNI coordinates } & \multirow[b]{2}{*}{ Cluster size } & \multirow[b]{2}{*}{ Peak $t$ value } \\
\hline & & & $\mathbf{x}$ & $\mathbf{Y}$ & $\mathbf{Z}$ & & \\
\hline \multirow[t]{7}{*}{$\mathrm{HC}>$ Smoker } & Inferior semi-lunar lobule & - & -9 & -72 & -51 & 660 & 6.25 \\
\hline & Cuneus & $18 / 30$ & 12 & -81 & 24 & 502 & 5.71 \\
\hline & Postcentral gyrus & 5 & 30 & -36 & 69 & 130 & 5.16 \\
\hline & Inferior frontal gyrus & 46 & 51 & 33 & 6 & 62 & 4.9 \\
\hline & Superior frontal gyrus & $8 / 9$ & -12 & 51 & 30 & 90 & 4.87 \\
\hline & Medial frontal gyrus & 8 & 15 & 42 & 33 & 74 & 4.77 \\
\hline & Insula & 13 & 48 & -9 & -12 & 69 & 4.4 \\
\hline \multirow[t]{2}{*}{$\mathrm{HC}<\mathrm{Smoker}$} & Orbitofrontal gyrus & 11 & 0 & 39 & -24 & 141 & 6.19 \\
\hline & Inferior temporal gyrus & 20 & 51 & -15 & -39 & 60 & 5.72 \\
\hline
\end{tabular}

ReHo, regional homogeneity; MNI, Montreal Neurological Institute; BA, Brodmann's area; HC, healthy controls. 
TABLE 3 | Brain regions showing significant differences in dynamic ALFF between groups.

\begin{tabular}{|c|c|c|c|c|c|c|c|}
\hline & \multirow[b]{2}{*}{ Brain regions } & \multirow[b]{2}{*}{ BA } & \multicolumn{3}{|c|}{ MNI coordinates } & \multirow[b]{2}{*}{ Cluster size } & \multirow[b]{2}{*}{ Peak $t$ value } \\
\hline & & & $\mathbf{x}$ & $\mathbf{Y}$ & $\mathbf{Z}$ & & \\
\hline \multirow[t]{2}{*}{$\mathrm{HC}<$ Smoker } & Inferior temporal gyrus & 21 & 57 & -18 & -27 & 50 & 4.82 \\
\hline & Anterior cingulate cortex & 32 & 15 & 45 & -12 & 141 & 4.72 \\
\hline
\end{tabular}

ALFF, amplitude of low frequency fluctuation; MNI, Montreal Neurological Institute; BA, Brodmann's area; HC, healthy controls.
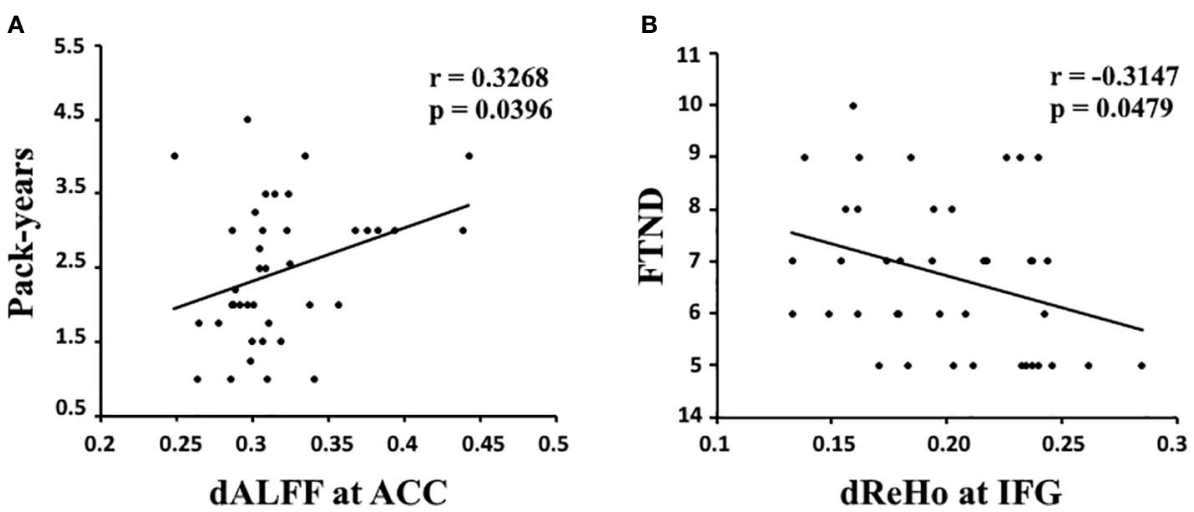

FIGURE 2 | (A) The dALFF variability in the ACC was positively correlated with pack-years in young smokers $(r=0.3268, p=0.0396$, uncorrected). (B) The dReHo variability in the IFG was negatively correlated with FTND scores in young smokers ( $r=-0.3147, p=0.0479$, uncorrected). dALFF, dynamic amplitude of lowfrequency fluctuation; dReHo, dynamic regional homogeneity; ACC, anterior cingulate cortex; IFG, inferior frontal gyrus; FTND, Fagerström Test for Nicotine dependence. Pack-years of smoking were calculated by multiplying the average number of packs of cigarettes smoked per day by the number of years the participant smoked.

monetary gains. They speculated that monetary rewards may be devalued relative to drug-related reward, and such aberrant reward function may be related to abnormal goal-directed behavior (55). In the context of nicotine addiction, studies have shown that nicotine-related stimuli can elicit overvalued rewards than nonnicotine stimuli in abstinent smokers. This finding may reflect that the ACC would assign a larger reward value to nicotine-related behaviors in a state of craving $(56,57)$. The current findings of increased ALFF temporal variability in ACC further confirmed the abnormal function of the ACC in young smokers. Considering the importance of ACC in evaluating reward value related to goal-directed behaviors, we tend to speculate that the abnormal temporal characteristics of dALFF in the ACC might reflect reward processing impairment in young smokers, which may facilitate action-selection mechanisms to converge on nicotine addiction.

Substantial evidence demonstrated that the ACC and prefrontal regions are primary nodes of a putative "cognitive control network" (58). Specifically, the ACC plays an important role in detecting salient events, especially to erroneous or errorprone actions (59). Once detecting such events or actions, the ACC further signals reorientation of attention implemented by the prefrontal regions (60). Previous studies have indicated that abnormalities in the ACC and prefrontal regions were associated with impaired cognitive function (executive, inhibitory, and decision-making) in smokers $(61,62)$. Abnormal neural activity in these regions in smokers during abstinence may suggest inefficient cognitive control in abstinent smokers (63). Taken together, the abnormal dALFF and dReHo in the ACC and prefrontal regions and correlations between the changes in dynamic regional indexes and smoking behavior may provide novel neuroimaging biomarkers of smoking in young smokers.

The current study has several limitations. First, the sample size was small. Larger samples will be needed in future studies. Second, physiological noise from respiratory cycles and cardiac activity were not monitored during fMRI scanning. Finally, the current findings are cross-sectional, and longitudinal studies are needed to elucidate the dynamic characteristics of regional neural activity.

\section{CONCLUSION}

To the best of our knowledge, very few studies have tried to assess dynamic regional neural activity in young smokers. We found increased dynamic ALFF variability in the ACC and decreased ReHo variability in frontal regions. Correlation analyses showed that the changes were related to the severity of nicotine addiction. This finding is indicative of the importance of cingulate and frontal regions in nicotine addiction in young smokers. Dynamic regional indexes might be used as a powerful supplement to static regional indexes, helping us obtain a more comprehensive understanding of neural activity in young smokers. 


\section{DATA AVAILABILITY STATEMENT}

The datasets presented in this article are not readily available because of the privacy of all the participants. Requests to access the datasets should be directed to fmydh@imust.edu.cn.

\section{ETHICS STATEMENT}

The studies involving human participants were reviewed and approved by Ethical Committee of medical research in First Affiliated Hospital of Baotou Medical College, Inner Mongolia University of Science and Technology. Written informed consent to participate in this study was provided by the participants' legal guardian/next of kin.

\section{AUTHOR CONTRIBUTIONS}

TX, DY, and KY were responsible for the study concept and design. FD, RH, YH, MZ, ZT, JT, XL, HJ, and YC contributed to the acquisition of fMRI data. TX and RH performed the data

\section{REFERENCES}

1. Yu D, Yuan K, Zhang B, Liu J, Dong M, Jin C, et al. White matter integrity in young smokers: a tract-based spatial statistics study. Addict Biol (2016) 21 (3):679-87. doi: 10.1111/adb.12237

2. National Center for Chronic Disease, P., S. Health Promotion Office on, and Health, Reports of the Surgeon General, in Preventing Tobacco Use Among Youth and Young Adults: A Report of the Surgeon General. Atlanta (GA): Centers for Disease Control and Prevention (US) (2012).

3. White HR, Bray BC, Fleming CB, Catalano RF. Transitions into and out of light and intermittent smoking during emerging adulthood. Nicotine Tob Res (2009) 11(2):211-9. doi: 10.1093/ntr/ntn017

4. Yuan K, Yu D, Zhao M, Li M, Wang R, Li Y, et al. Abnormal frontostriatal tracts in young male tobacco smokers. NeuroImage (2018) 183:346-55. doi: 10.1016/j.neuroimage.2018.08.046

5. Su S, Yu D, Cheng J, Chen Y, Zhang X, Guan Y, et al. Decreased Global Network Efficiency in Young Male Smoker: An EEG Study during the Resting State. Front Psychol (2017) 8(1605). doi: 10.3389/fpsyg.2017.01605

6. Bi Y, Yuan K, Yu D, Wang R, Li M, Li Y, et al. White matter integrity of central executive network correlates with enhanced brain reactivity to smoking cues. Hum Brain Mapp (2017) 38(12):6239-49. doi: 10.1002/ hbm. 23830

7. Zhao M, Liu J, Cai W, Li J, Zhu X, Yu D, et al. Support vector machine based classification of smokers and nonsmokers using diffusion tensor imaging. Brain Imaging Behav (2019). doi: 10.1007/s11682-019-00176-7

8. Yuan K, Yu D, Bi Y, Wang R, Li M, Zhang Y, et al. The left dorsolateral prefrontal cortex and caudate pathway: New evidence for cue-induced craving of smokers. Hum Brain Mapp (2017) 38(9):4644-56. doi: 10.1002/ hbm. 23690

9. Biswal B, Zerrin Yetkin F, Haughton VM, Hyde JS. Functional connectivity in the motor cortex of resting human brain using echo-planar mri. Magn Reson Med (1995) 34(4):537-41. doi: 10.1002/mrm.1910340409

10. Yuan K, Yu D, Bi Y, Li Y, Guan Y, Liu J, et al. The implication of frontostriatal circuits in young smokers: A resting-state study. Hum Brain Mapp (2016) 37(6):2013-26. doi: 10.1002/hbm.23153 analysis. DY and KY provided critical revision of the manuscript for important intellectual context.

\section{FUNDING}

This work is supported by the National Natural Science Foundation of China under Grant Nos. 81871430, 81871426, $61771266,81401488,81701780,31800926,81401478$, 81401488,81470816 , and 81471737 , the program for Young Talents of Science and Technology in Universities of Inner Mongolia Autonomous Region NJYT-17-B11, the Natural Science Foundation of Inner Mongolia under Grant No. 2019JQ07, the science and technology planning project of Inner Mongolia Autonomous Region 2019GG109, the Chunhui Program of the Ministry of Education of the People's Republic of China 2018-45, the Fundamental Research Funds for the Central Universities under Grant No. JB151204, the Natural Science Basic Research Plan in Shaan-xi Province of China under Grant No. 2018JM7075, and the US National Institutes of Health, Intramural Research program Y1AA3009.

11. Yu D, Yuan K, Bi Y, Luo L, Zhai J, Liu B, et al. Altered interhemispheric resting-state functional connectivity in young male smokers. Addict Biol (2018) 23(2):772-80. doi: 10.1111/adb.12515

12. Yu R, Zhao L, Tian J, Qin W, Wang W, Yuan K, et al. Regional homogeneity changes in heavy male smokers: a resting-state functional magnetic resonance imaging study. Addict Biol (2013) 18(4):729-31. doi: 10.1111/j.13691600.2011.00359.x

13. Bu L, Yu D, Su S, Ma Y, von Deneen KM, Luo L, et al. Functional Connectivity Abnormalities of Brain Regions with Structural Deficits in Young Adult Male Smokers. Front Hum Neurosci (2016) 10(494). doi: 10.3389/fnhum.2016.00494

14. Zhao S, Li Y, Li M, Wang R, Bi Y, Zhang Y, et al. 12-h abstinence-induced functional connectivity density changes and craving in young smokers: a resting-state study. Brain Imaging Behav (2019) 13(4):953-62. doi: 10.1007/ s11682-018-9911-3

15. Wang C, Bai J, Wang C, von Deneen KM, Yuan K, Cheng J. Altered thalamocortical resting state functional connectivity in smokers. Neurosci Lett (2017) 653:120-5. doi: 10.1016/j.neulet.2017.05.038

16. Li Y, Yuan K, Guan Y, Cheng J, Bi Y, Shi S, et al. The implication of salience network abnormalities in young male adult smokers. Brain Imaging Behav (2017) 11(4):943-53. doi: 10.1007/s11682-016-9568-8

17. Bi Y, Yuan K, Guan Y, Cheng J, Zhang Y, Li Y, et al. Altered resting state functional connectivity of anterior insula in young smokers. Brain Imaging Behav (2017) 11 (1):155-65. doi: 10.1007/s11682-016-9511-z

18. Raichle ME. The Brain's Dark Energy. Science (2006) 314(5803):1249-50. doi: $10.1126 /$ science. 1134921

19. Fox MD, Raichle ME. Spontaneous fluctuations in brain activity observed with functional magnetic resonance imaging. Nat Rev Neurosci (2007) 8 (9):700-11. doi: 10.1038/nrn2201

20. Zang Y, Jiang T, Lu Y, He Y, Tian L. Regional homogeneity approach to fMRI data analysis. NeuroImage (2004) 22(1):394-400. doi: 10.1016/j.neuroimage. 2003.12.030

21. Zang Y, He Y, Zhu C, Cao Q, Sui M, Liang M, et al. Altered baseline brain activity in children with ADHD revealed by resting-state functional MRI. Brain Dev (2007) 29(2):83-91. doi: 10.1016/j.braindev.2006.07.002 
22. Qiu Y, Han L, Lv X, Jiang G, Tian J, Zhuo F, et al. Regional Homogeneity Changes in Heroin-dependent Individuals: Resting-State Functional MR Imaging Study. Radiology (2011) 261(2):551-9. doi: 10.1148/radiol. 11102466

23. Liu F, Guo W, Liu L, Long Z, Ma C, Xue Z, et al. Abnormal amplitude lowfrequency oscillations in medication-naive, first-episode patients with major depressive disorder: A resting-state fMRI study. J Affect Disord (2013) 146 (3):401-6. doi: 10.1016/j.jad.2012.10.001

24. He Y, Wang L, Zang Y, Tian L, Zhang X, Li K, et al. Regional coherence changes in the early stages of Alzheimer's disease: A combined structural and resting-state functional MRI study. NeuroImage (2007) 35(2):488-500. doi: 10.1016/j.neuroimage.2006.11.042

25. Tang J, Liao Y, Deng Q, Liu T, Chen X, Wang X, et al. Altered spontaneous activity in young chronic cigarette smokers revealed by regional homogeneity. Behav Brain Funct (2012) 8(1):44. doi: 10.1186/1744-9081-8-44

26. Chen H, Mo S. Regional Homogeneity Changes in Nicotine Addicts by Resting-State fMRI. PloS One (2017) 12(1):e0170143-e0170143. doi: 10.1371/journal.pone. 0170143

27. Wang C, Shen Z, Huang P, Yu H, Qian W, Guan X, et al. Altered spontaneous brain activity in chronic smokers revealed by fractional ramplitude of lowfrequency fluctuation analysis: a preliminary study. Sci Rep (2017) 7(1):328. doi: 10.1038/s41598-017-00463-7

28. Feng D, Yuan K, Li Y, Cai C, Yin J, Bi Y, et al. Intra-regional and inter-regional abnormalities and cognitive control deficits in young adult smokers. Brain Imaging Behav (2016) 10(2):506-16. doi: 10.1007/s11682-015-9427-z

29. Hindriks R, Adhikari MH, Murayama Y, Ganzetti M, Mantini D, Logothetis NK, et al. Corrigendum to "Can sliding-window correlations reveal dynamic functional connectivity in resting-state fMRI?" [NeuroImage 127 (2016) 242256]. NeuroImage (2016) 132:115-5. doi: 10.1016/j.neuroimage.2016.02.007

30. Hudetz AG, Liu X, Pillay S. Dynamic repertoire of intrinsic brain states is reduced in propofol-induced unconsciousness. Brain Connect (2015) 5(1):1022. doi: $10.1089 /$ brain.2014.0230

31. Douw L, Wakeman DG, Tanaka N, Liu H, Stufflebeam SM. State-dependent variability of dynamic functional connectivity between frontoparietal and default networks relates to cognitive flexibility. Neuroscience (2016) 339:1221. doi: 10.1016/j.neuroscience.2016.09.034

32. Lin S, Vavasour I, Kosaka B, Li DKB, Traboulsee A, MacKay A, et al. Education, and the balance between dynamic and stationary functional connectivity jointly support executive functions in relapsing-remitting multiple sclerosis. Hum Brain Mapp (2018) 39(12):5039-49. doi: 10.1002/hbm.24343

33. Espinoza FA, Liu J, Ciarochi J, Turner JA, Vergara VM, Caprihan A, et al. Dynamic functional network connectivity in Huntington's disease and its associations with motor and cognitive measures. Hum Brain Mapp (2019) 40 (6):1955-68. doi: $10.1002 / \mathrm{hbm} .24504$

34. Liu F, Wang Y, Li M, Wang W, Li R, Zhang Z, et al. Dynamic functional network connectivity in idiopathic generalized epilepsy with generalized tonic-clonic seizure. Hum Brain Mapp (2017) 38(2):957-73. doi: 10.1002/ hbm. 23430

35. Fu Z, Tu Y, Di X, Du Y, Pearlson GD, Turner JA, et al. Characterizing dynamic amplitude of low-frequency fluctuation and its relationship with dynamic functional connectivity: An application to schizophrenia. NeuroImage (2018) 180:619-31. doi: 10.1016/j.neuroimage.2017.09.035

36. Liao X, Yuan L, Zhao T, Dai Z, Shu N, Xia M, et al. Spontaneous Functional Network Dynamics and Associated Structural Substrates in the Human Brain. Front Hum Neurosci (2015) 9(478). doi: 10.3389/fnhum.2015.00478

37. Cui Q, Sheng W, Chen Y, Pang Y, Lu F, Tang Q, et al. Dynamic changes of amplitude of low-frequency fluctuations in patients with generalized anxiety disorder. Hum Brain Mapp (2020) 41(6):1667-76. doi: 10.1002/hbm.24902

38. Lottman K, Kraguljac N, White D, Morgan C, Calhoun V, Butt A, et al. Risperidone Effects on Brain Dynamic Connectivity- a Prospective Resting State fMRI Study in Schizophrenia. Front Psychiatry (2017) 8(14). doi: 10.3389/fpsyt.2017.00014

39. Fagerstrom KO, Schneider NG. Measuring nicotine dependence: A review of the Fagerstrom Tolerance Questionnaire. J Behav Med (1989) 12(2):159-82. doi: $10.1007 / \mathrm{BF} 00846549$

40. Heatherton TF, Kozlowski LT, Frecker RC, Fagerstrom KO. The Fagerström Test for Nicotine Dependence: a revision of the Fagerstrom Tolerance Questionnaire. Br J Addict (1991) 86(9):1119-27. doi: 10.1111/j.1360-0443.1991.tb01879.x
41. McBride D, Barrett SP, Kelly JT, Aw A, Dagher A. Effects of Expectancy and Abstinence on the Neural Response to Smoking Cues in Cigarette Smokers: an fMRI Study. Neuropsychopharmacology (2006) 31(12):2728-38. doi: 10.1038/ sj.npp. 1301075

42. Yan C, Yang Z, Colcombe SJ, Zuo X, Milham MP. Concordance among indices of intrinsic brain function: Insights from inter-individual variation and temporal dynamics. Sci Bull (2017) 62(23):1572-84. doi: 10.1016/ j.scib.2017.09.015

43. Thompson GJ, Magnuson ME, Merritt MD, Schwarb H, Pan W, McKinley A, et al. Short-time windows of correlation between large-scale functional brain networks predict vigilance intraindividually and interindividually. Hum Brain Mapp (2013) 34(12):3280-98. doi: 10.1002/hbm.22140

44. Gonzalez Castillo J, Hoy CW, Handwerker DA, Robinson ME, Buchanan LC, Saad ZS, et al. Tracking ongoing cognition in individuals using brief, wholebrain functional connectivity patterns. Proc Natl Acad Sci (2015) 112 (28):8762-7. doi: 10.1073/pnas.1501242112

45. Chen J, Sun D, Shi Y, Jin W, Wang Y, Xi Q, et al. Dynamic Alterations in Spontaneous Neural Activity in Multiple Brain Networks in Subacute Stroke Patients: A Resting-State fMRI Study. Front Neurosci (2019) 12(994). doi: 10.3389/fnins.2018.00994

46. Sakoğlu Ü, Pearlson GD, Kiehl KA, Wang Y, Michael AM, Calhoun VC. A method for evaluating dynamic functional network connectivity and taskmodulation: application to schizophrenia. Magn Reson Mater Phys Biol Med (2010) 23(5):351-66. doi: 10.1007/s10334-010-0197-8

47. Leonardi N, Van De Ville D. On spurious and real fluctuations of dynamic functional connectivity during rest. NeuroImage (2015) 104:430-6. doi: 10.1016/j.neuroimage.2014.09.007

48. Allen EA, Damaraju E, Plis SM, Erhardt EB, Eichele T, Calhoun VD. Tracking Whole-Brain Connectivity Dynamics in the Resting State. Cereb Cortex (2012) 24(3):663-76. doi: 10.1093/cercor/bhs352

49. Volkow ND, Fowler JS, Wang GJ, Swanson JM, Telang F. Dopamine in Drug Abuse and Addiction: Results of Imaging Studies and Treatment Implications. Arch Neurol (2007) 64(11):1575-9. doi: 10.1001/archneur.64.11.1575

50. Yuan K, Zhao M, Yu D, Manza P, Volkow ND, Wang GJ, et al. Striato-cortical tracts predict 12-h abstinence-induced lapse in smokers. Neuropsychopharmacology (2018) 43(12):2452-8. doi: 10.1038/s41386-018-0182-x

51. Hyman SE, Malenka RC, Nestler EJ. Neural Mechanisms of Addiction: The Role of Reward-Related Learning and Memory. Annu Rev Neurosci (2006) 29 (1):565-98. doi: 10.1146/annurev.neuro.29.051605.113009

52. Peoples LL. Will, Anterior Cingulate Cortex, and Addiction. Science (2002) 296(5573):1623-4. doi: 10.1126/science.1072997

53. Goldstein RZ, Tomasi D, Rajaram S, Cottone LA, Zhang L, Maloney T, et al. Role of the anterior cingulate and medial orbitofrontal cortex in processing drug cues in cocaine addiction. Neuroscience (2007) 144(4):1153-9. doi: 10.1016/j.neuroscience.2006.11.024

54. Holroyd CB, Yeung N. Motivation of extended behaviors by anterior cingulate cortex. Trends Cogn Sci (2012) 16(2):122-8. doi: 10.1016/j.tics. 2011.12.008

55. Baker TE, Stockwell T, Barnes G, Haesevoets R, Holroyd CB. Reward Sensitivity of ACC as an Intermediate Phenotype between DRD4-521T and Substance Misuse. J Cogn Neurosci (2016) 28(3):460-71. doi: 10.1162/ jocn_a_00905

56. Baker TE, Lesperance P, Tucholka A, Potvin S, Larcher K, Zhang Y, et al. Reversing the Atypical Valuation of Drug and Nondrug Rewards in Smokers Using Multimodal Neuroimaging. Biol Psychiatry (2017) 82(11):819-27. doi: 10.1016/j.biopsych.2017.01.015

57. Baker TE, Wood JMA, Holroyd CB. Atypical valuation of monetary and cigarette rewards in substance dependent smokers. Clin Neurophysiol (2016) 127(2):1358-65. doi: 10.1016/j.clinph.2015.11.002

58. Sutherland MT, McHugh MJ, Pariyadath V, Stein EA. Resting state functional connectivity in addiction: Lessons learned and a road ahead. NeuroImage (2012) 62(4):2281-95. doi: 10.1016/j.neuroimage.2012.01.117

59. Carter CS, Veen VV. Anterior cingulate cortex and conflict detection: An update of theory and data. Cogn Affect Behav Neurosci (2007) 7(4):367-79. doi: 10.3758/CABN.7.4.367

60. Kerns JG, D.Cohen J, III AWM, Cho RY, Stenger VA, Carter CS. Anterior Cingulate Conflict Monitoring and Adjustments in Control. Science (2004) 303(5660):1023-6. doi: 10.1126/science.1089910 
61. Li Y, Yuan K, Cai C, Feng D, Yin J, Bi Y, et al. Reduced frontal cortical thickness and increased caudate volume within fronto-striatal circuits in young adult smokers. Drug Alcohol Depend (2015) 151:211-9. doi: 10.1016/ j.drugalcdep.2015.03.023

62. Hayashi T, Ko JH, Strafella AP, Dagher A. Dorsolateral prefrontal and orbitofrontal cortex interactions during self-control of cigarette craving. Proc Natl Acad Sci (2013) 110(11):4422-7. doi: 10.1073/pnas.1212185110

63. Sweitzer MM, Geier CF, Joel DL, McGurrin P, Denlinger RL, Forbes EE, et al. Dissociated Effects of Anticipating Smoking versus Monetary Reward in the Caudate as a Function of Smoking Abstinence. Biol Psychiatry (2014) 76 (9):681-8. doi: 10.1016/j.biopsych.2013.11.013
Conflict of Interest: The authors declare that the research was conducted in the absence of any commercial or financial relationships that could be construed as a potential conflict of interest.

Copyright (C) 2020 Xue, Dong, Huang, Tao, Tang, Cheng, Zhou, Hu, Li, Yu, Ju and Yuan. This is an open-access article distributed under the terms of the Creative Commons Attribution License (CC BY). The use, distribution or reproduction in other forums is permitted, provided the original author(s) and the copyright owner(s) are credited and that the original publication in this journal is cited, in accordance with accepted academic practice. No use, distribution or reproduction is permitted which does not comply with these terms. 